\title{
INHALT
}

\section{Das Strafprozeßrecht}

im Rahmen der gesamten Strafrechtspflege

1. Vorbemerkung ............. . 15

1.1. Begriff des Strafprozeßrechts . . . . . . . . 15

1.2. Einbettung in die Gesamtkultursituation . . . . . 15

1.3. Einordnung in das Verfassungsrecht . . . . . . . 16

1.4. Justizmonopol und Justizgewährungspflicht des Staates 17

2. Strafrecht und Strafprozeßredit . . . . . . . . 18

2.1. Die Realisierung des staatlichen Strafanspruchs .. 18

2.2. Die Aufgaben des Strafrichters . . . . . . . . 18

2.3. Materielles und formelles Recht als Wirkungseinheit . 19

3. Strafverfahrensredht und Kriminologie ....... 20

II. Die Organe der Strafrechtspflege

und die sonstigen Verfahrensbeteiligten

1. Das Gericht . . . . . . . . . . . . .

1.1. Die Bedeutung des unabhängigen Richters für die Strafrechtspflege ............ 21

1.2. Die Mitwirkung von Laienrichtern . . . . . . . 22

1.3. Die Gerichtsverfassung im Bereich der Strafjustiz . . 23

1.4. Die Zuständigkeit ............. . . 24

1.41. Die örtliche Zuständigkeit (Gerichtsstand) . . 24

1.42. Die sachliche Zuständigkeit . . . . . . . . 28

1.5. Der gesetzliche Richter . . . . . . . . . . . 31

1.51. Unzulässigkeit von Ausnahmegerichten ... 32

1.52. Die Bestimmung des gesetzlichen Richters . . 32

1.6. Der befangene Strafrichter ........... 35

1.61. Der ausgeschlossene Strafrichter . . . . . . 36

1.62. Der ablehnbare Strafrichter . . . . . . 37

1.7. Der Urkundsbeamte als Hilfsorgan des Gerichts . . 41

2. Die Staatsanwaltschaft ............. 41

2.1. Historische Entstehung und grundlegende Bedeutung . 41 
2.2. Die Rechtsstellung der Staatsanwaltschaft und ihre Einordnung in die Staatsgewalt . . . . . . .

2.21. Das Verhältnis der Staatsanwaltschaft zur inneren Verwaltung ........... 43

2.22. Das Verhältnis der Staatsanwaltschaft zur Justiz 44

2.23. Bestimmung der Rechtsstellung der Staatsanwaltschaft im einzelnen .......... 44

2.3. Aufbau und Organisation der Staatsanwaltschaft . . 45

2.31. Hierarchischer Behördenaufbau . . . . . . 45

2.32. Organisation der Staatsanwaltschaft . . . . . 45

2.4. Die Aufgaben der Staatsanwaltschaft im Überblick . 46

3. Der Verteidiger . . . . . . . . . . . 47

3.1. Aufgabe und Stellung des Verteidigers im Strafverfahren . . . . . . . . . . . 47

3.2. Das Verhältnis zum Beschuldigten . . . . . . 49

3.3. Die Befugnisse des Verteidigers (Uberblidk) . . . . 49

3.31. Ungehinderter Verkehr mit dem Beschuldigten . $\quad 49$

3.32. Anwesenheit und Mitwirkung bei Verfahrenshandlungen .......... . . 50

3.33. Das Recht auf Akteneinsicht . . . . . . 50

3.4. Die notwendige Verteidigung ........ 51

3.5. Wahlverteidiger und Offizialverteidiger . . . . 53

3.6. Der Ausschluß des Verteidigers . . . . . . . . . . 54

3.7. Der Beistand ... . . . . . . . . . 55

4. Die sonstigen Verfahrensbeteiligten ......... . 56

4.1. Der Beschuldigte .. . . . . . . . . . . . 56

4.11. Die Rechtsstellung des Beschuldigten . . . . . 56

4.12. Die Rechte des Beschuldigten ....... . 56

4.13. Pflichten des Beschuldigten . . . . . . . . 58

4.2. Der Verletzte . . . . . . . . . . . . . . 59

4.21. Begriff des Verletzten . . . . . . . . 59

4.22. Die Beteiligung des Verletzten am Strafverfahren .............. 60

4.3. Nebenbeteiligte (Überblidk) . . . . . . . 63

III. Die allgemeinen Verfahrensgrundsätze

1. Vorbemerkung . . . . . . . . . . . 64

2. Die einzelnen Prinzipien ........... . 64 
2.1. Das Offizialprinzip ............ . . 64

2.11. Inhalt und Ausgestaltung in der StPO . . . . 64

2.12. Ausnahmen und Einschränkungen des Offizial- 65 prinzips ...........

2.2. Das Akkusationsprinzip . . . . . . . . . . . . . 66

2.21. Die grundsätzliche Bedeutung ...... 66

2.22. Die Ausgestaltung im geltenden Recht . . . . 67

2.3. Das Legalitäts- und das Opportunitätsprinzip . . . 69

2.31. Begriffe und Bedeutung ......... 69

2.32. Die einzelnen Einstellungsmöglichkeiten nach den

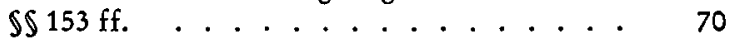

2.33. Die Sicherung des Legalitätsprinzips durch das Klageerzwingungsverfahren ...... 77

2.4. Die Instruktionsmaxime . . . . . . . . . 80

2.5. Das Beschleunigungsgebot .......... 81

2.6. Die prozessuale Fürsorgepflicht . . . . . . . 82

IV. Der Ablauf des Strafverfahrens

1. Allgemeine Fragen des Verfahrensganges ...... 84

1.1. Vorbemerkung . . . . . . . . . . . 84

1.2. Die prozessualen Handlungen der Verfahrensbeteiligten 84

1.21. Die Prozeßhandlungen im allgemeinen . . . . 84

1.22. Die richterlichen Entscheidungen . . . . . . 91

1.3. Die Verfahrensvoraussetzungen ........ 92

1.31. Bedeutung und Funktion ......... 92

1.32. Uberblick über die wichtigsten Verfahrensvoraussetzungen ........... . . 93

1.33. Die prozessuale Behandlung . . . . . . . 94

1.4. Der Verfahrensgegenstand . . . . . . . . . 95

1.41. Praktische Bedeutung . . . . . . . . . 95

1.42. Die Abhängigkeit des Verfahrensgegenstandes vom Prozeßablauf . . . . . . . . . 95

1.43. Der dogmatische Aufbau des Verfahrensgegenstandes . . . . . . . . . . . . 98

1.44. Zusammenfassung mehrerer Strafsachen in einem Verfahren . . . . . . . . . . 99

1.5. Fristen und Termine . . . . . . . . . 100

1.51. Begriff und Funktion . . . . . . . . 100

1.52. Die Wiedereinsetzung in den vorigen Stand bei einer Fristversäumung . . . . . . . . 101 
1.6. Überblick über die einzelnen Abschnitte des Strafverfahrens . . . . . . . . . . . 103

2. Das Vorverfahren .............. 104

2.1. Aufgabe und Ziel des Ermittlungsverfahrens . . . . 104

2.2. Die Träger des Ermittlungsverfahrens . . . . . . . 105

2.21. Staatsanwaltschaft und Polizei . . . . . . 105

2.22. Kompetenzabgrenzung . . . . . . . . . . 105

2.3. Der Beschuldigte und seine Rechtsstellung . . . . . 107

2.31. Der Begriff des Beschuldigten . . . . . . . . 107

2.32. Rechte und Pflichten des Beschuldigten ... . 108

2.33. Die Funktion des Verteidigers im Ermittlungsverfahren .......... 110

2.4. Der Gang des Ermittlungsverfahrens . . . . . . 110

2.41. Die Einleitung des Ermittlungsverfahrens . . . 110

2.42. Die Durchführung der Ermittlungen . . . . . 111

2.5. Zwangsmaßnahmen im Zuge der Ermittlungen . . . 112

2.51. Vorbemerkung . . . . . . . . . . . . . 112

2.52. Die Untersuchungshaft . . . . . . . . . . 113

2.53. Die Beschlagnahme . . . . . . . . . 125

2.6. Der Abschluß des Ermittlungsverfahrens . . . . . 127

2.61. Die Einstellung des Verfahrens . . . . . . . 127

2.62. Die Erhebung der öffentlichen Klage . . . . . 129

2.7. Die gerichtliche Voruntersuchung . . . . . . . . . 132

2.71. Bedeutung . . . . . . . . . . . . 132

2.72. Die Voraussetzungen der gerichtlichen Voruntersuchung ............. . . 133

2.73. Die Aufgaben des Untersuchungsrichters . . 134

3. Das Zwischenverfahren . . . . . . . . . . . . . 137

3.1. Aufgabe und Bedeutung . . . . . . . . . . . 137

3.2. Vorbereitung der Entscheidung im Eröffnungsverfahren ................ 138

3.3. Die gerichtliche Entscheidung . . . . . . . . 139

3.31. Zulassung der Anklage .... . . . . . . . . 139

3.32. Der Nichteröffnungsbeschluß . . . . . . 140

3.33. Vorläufige Einstellung . . . . . . . . . . . 141

3.4. Anklage und Eröffnungsbeschluß im beschleunigten

Verfahren ........... 142

4. Das Hauptverfahren ............. 142

4.1. Die Vorbereitung der Hauptverhandlung . . . . . 142

4.2. Die Durchführung der Hauptverhandlung . . . . . 143 
4.21. Die Verfahrensgrundsätze zur Durchführung der Hauptverhandlung

4.22. Der Ablauf der Hauptverhandlung . . . . . 150

4.23. Die Abschnitte der Hauptverhandlung im Oberblidk

4.3. Die Beweisaufnahme ........... 158

4.31. Der Begriff des Beweises ......... . 158

4.32. Allgemeine Fragen des Beweisrechts . . . . . 160

4.33. Die Beweismittel . . . . . . . . 161

4.34. Beweisverbote . . . . . . . . . . . 177

4.35. Die Durchführung der Beweisaufnahme . . . 179

4.36. Die Beweiswürdigung . . . . . . . . 185

4.4. Das Urteil in Strafsachen . . . . . . . . . . . 188

4.41. Die Abfassung und Verkündung des Urteils . . 188

4.42. Der Urteilstenor . . . . . . . . . . . . 189

4.43. Die Urteilsgründe . . . . . . . . . . . . . 191

4.5. Die Rechtskraft . . . . . . . . . . . . 194

4.51. Formelle und materielle Rechtskraft . . . . . 194

4.52. Durchbrechung der Rechtskraft . . . . . . . 196

4.53. Die Teilrechtskraft . . . . . . . . . 196

5. Das Rechtsmittelverfahren . . . . . . . . . . 197

5.1. Allgemeine Fragen der Rechtsmitteleinlegung . . . 197

5.11. Die rechtspolitische Notwendigkeit eines Instanzenzuges . . . . . . . . . . . . 197

5.12. Arten der Rechtsmittel . . . . . . . . . . 198

5.13. Allgemeine Voraussetzungen der Rechtsmitteleinlegung .............. . . 198

5.14. Allgemeine Wirkungen der Rechtsmitteleinlegung 199

5.15. Der Umfang der Anfechtung . . . . . . 199

5.16. Das Verschlechterungsverbot . . . . . . . 201

5.17. Die Rechtsmittelerklärung . . . . . . . 201

5.2. Die Berufung ... . . . . . . . . . 203

5.21. Begriff und Voraussetzungen . . . . . . . 203

5.22. Die Berufungsverhandlung . . . . . . 203

5.23. Die Entscheidung des Berufungsgerichts . . 204

5.3. Die Revision . . . . . . . . . . 205

5.31. Formelle Voraussetzungen . . . . . . 205

5.32. Das Verfahren in der Revisionsinstanz . . . . 210

5.33. Die Entscheidung des Revisionsgerichts . . . 211

5.4. Die Beschwerde . . . . . . . . . . 213

5.41. Arten der Beschwerde . . . . . . . . 213 
5.42. Das Beschwerdeverfahren ........ 213

5.43. Die Entscheidung des Beschwerdegerichts . . . 213

5.5. Die Wiederaufnahme . . . . . . . . . 214

5.51. Rechtsnatur . . . . . . . . . 214

5.52. Die Voraussetzungen des Wiederaufnahmeverfahrens ........... 214

5.53. Das Zulassungsverfahren . . . . . . . . . . . 215

5.54. Die neue Hauptverhandlung . . . . . . . . 215

6. Die Kosten des Verfahrens . . . . . . . . . . . . . 215

7. Die Strafvollstredkung . . . . . . . . . . . 216

V. Besondere Verfahrensarten

1. Die Privatklage . . . . . . . . . . . . . 217

1.1. Besonderheiten gegenüber der Offizialklage . . . . 217

1.2. Die Zulässigkeit der Privatklage . . . . . . . . . 218 1.21. Die Privatklagedelikte . . . . . . . . . 218

1.22. Weitere formelle Voraussetzungen . . . . . 218

1.3. Der Verfahrensgang . . . . . . . . . . . 218

1.31. Die Eröffnung des Verfahrens ...... . 218

1.32. Die Durchführung des Verfahrens . . . . . . 219

1.33. Die Widerklage . . . . . . . . . . . . 219

1.34. Die Beendigung des Privatklageverfahrens . . 219

2. Das Strafbefehlsverfahren .. . . . . . . . . 220

2.1. Bedeutung und Problematik . . . . . . . . 220

2.2. Der Erlaß des Strafbefehls . . . . . . . . . . . . 221

2.21. Der Antrag der Staatsanwaltschaft und die Entscheidung des Amtsrichters ....... . 221

2.22. Der Inhalt des Strafbefehls . . . . . . . . . 222

2.3. Der Einspruch und die Einspruchsverhandlung . . . 222

2.4. Die Rechtskraft des Strafbefehls . . . . . . . . . 223

3. Weitere Sonderformen des Verfahrens . . . . . . . . 224

Sadhregister . . . . . . . . . . . . . 225 